\title{
Some considerations for future research into the risks of radiation-induced cardiovascular diseases
}

\author{
Klaus Rüdiger Trott $^{1}$
}

Received: 22 July 2016 / Accepted: 26 July 2016 / Published online: 24 August 2016

(C) Springer-Verlag Berlin Heidelberg 2016

The radiosensitivity of the heart has been known since Louis Fajardo described severe myocardial fibrosis leading to congestive heart failure in patients who had been cured from Hodgkin's disease by mantle field radiotherapy $[1,2]$. In the classical form of this treatment, considerable parts of the heart are in the primary beam and receive doses over 40 Gy (fractionated). The report by Kodama in the early 1990s of a significant dose-related increase in deaths from heart diseases among Japanese A-bomb survivors observed in the Life Span Study, which was compatible with a linear non-threshold dose-risk relationship [3], stimulated a large number of epidemiological and clinical research activities, e. g. in patients treated for nonmalignant disorders such as peptic ulcer by Carr et al. [4] or in many other Hodgkin's disease cohorts [5-7]. The most important studies addressed the cardiovascular disease risk of patients who had been cured from breast cancer by postoperative radiotherapy [8, 9]. Other radiotherapy studies explored more specific heart diseases, such as myocardial infarction or valvular diseases $[10,11]$. An increased mortality from heart diseases was also demonstrated among people who had been occupationally exposed to ionizing radiation $[12,13]$. The results of these studies were reviewed again and again, by individual scientists or committees, e. g. [14, 15], thus confirming the extraordinary radiosensitivity of the heart. Most studies had very poor dosimetry, none had individual dosimetry and nearly all relied on mean heart dose estimates. Only few studies attempted to determine

Klaus Rüdiger Trott klaustrott@yahoo.it

1 UCL Cancer Institute, University College London, London, UK partial heart doses in relation to specific radiation-induced heart diseases [16-19].

The results of these studies were often considered by workshops, committees and institutions with a special interest in the potential implications they would have for the current system of radiation protection. The Article 31 Group of Experts of the Euratom Treaty discussed the problem in 2008 [20]. They concluded that there was convincing evidence for a significant risk of cardiovascular mortality after moderate and low radiation doses, and that policy and regulatory implication should be further discussed and research in this field stimulated. The number of publications, reviews and presentations at international conferences has increased rapidly. At present, it is nearly impossible to determine whether any new fundamental information has come from all the epidemiological work. However, there are several issues which call for caution in interpretation of the results of the plethora of epidemiological studies:

1. Radiation exposure has been proven to be able to cause five different heart diseases: pericarditis, myocardial fibrosis, myocardial infarction, conduction defects and valvular insufficiency. The anatomical site of the manifestation of the pathology is different for each of these heart diseases, and thus the location of the target within the organ is also likely to be different. As yet, no study has been published which addressed this fundamental problem. Particularly in medical exposures, where inhomogeneous dose distributions within the organ are a hallmark of exposure, the relationship between dose distribution within the organ and type and location of heart disease would be a high-priority research programme. Such studies would also help to identify radiobiological pathogenic pathways in humans, and provide sound criteria for optimizing treatment plans in radiation on- 
cology and setting standards of heart risk-related dose specification in diagnostic radiology.

2. Worldwide, the European Atomic Energy Community (EURATOM) has been at the forefront of developing and supporting research on the biological mechanisms underlying the development of pathological changes in the heart after low and intermediate radiation exposure. The CARDIORISK project [21] was the first international cooperative project in this field. It was coordinated by the department of radiation oncology of the Technical University Munich and ran from 2008 to 2011. More than 30 papers from the CARDIORISK project have been published in refereed journals so far. This approach was extended in the next EURATOM project PROCARDIO [22], coordinated by the Helmholtz Centre Munich, which started in 2012. A large amount of new information was accumulated in these large research projects which continue to publish. It goes without saying that any new research project into the biology, the mechanisms and the biological modelling of radiation-induced heart diseases and dose specification for epidemiological studies has to be based on the results of these EUfunded projects. Very little research into the radiobiology of radiation-induced heart diseases has been performed outside Europe.

3. Modelling approaches often fail to consider the fundamental difference between the radiation dose dependence of cancer risk and the radiation dose dependence of cardiovascular risk. Whereas cancer incidence or mortality rates increase with increasing dose, for cardiovascular diseases it is the severity of the disease and the rate of severity progression which depends on dose [23]. This problem is not easy to overcome but is usually washed away by using severity scoring systems which have been developed for completely different purposes and are more or less useless for research of the role of radiation in the development and progression of highly specific and multifactorial diseases such as radiation-induced heart disease. This causes particular problems in animal experiments using small rodents, but can be overcome in prospective clinical studies. Such a study should first determine a detailed three-dimensional-dose map of the heart (e. g. in postoperative radiotherapy of breast cancer), followed by repeated functional imaging (CardioMR, SPECT perfusion studies, clinical heart function studies etc.) at biennial intervals. The aim would be to study the localization of pathological and pathophysiological changes in relation to the anatomical dose distribution and the progression of these changes over the years (follow-up at least 10 years). Such a study is currently in progress at the department of radiation oncology of the Technical University Munich.
4. In vitro studies on established endothelial cell lines or primary cells which are irradiated and followed in vitro are of limited value for investigation of the pathogenic pathways leading to microvascular insufficiency and radiation-induced heart diseases. The experimental design developed by the CARDIORISK project overcomes many of the problems by giving high-precision local radiation exposure to the heart and keeping the animals until their natural end of life up to 12 months later. This way, cardiac pathology develops under physiological conditions, and additional stress factors or protective modulation can be added to study their effect in vivo. However, the possibilities for studying the pathophysiology of radiation-induced heart damage in rodents are limited. Therefore, methods have been developed in the CARDIORISK project to study changes-from organ physiology to tissue pathology and molecular biology of different cell types-developing in a physiological tissue environment many months after exposure. Of particular interest are the long-term changes in the biology and molecular biology of microvascular endothelial cells and cardiomyocytes. Significant late and very late changes after doses well below 1 Gy have been observed both in the mitochondria of cardiomyocytes [24] and in microvascular endothelial cells [25].

5. Dosimetric studies in patients demonstrated the pronounced interindividual variations of dose distributions which are entirely due to differences in patient anatomy $[17,19,26]$. In order to develop a sound understanding of the dose dependence of radiation-induced cardiovascular diseases, prospective epidemiological studies with precise dosimetry concentrating on local dose distribution are required. Very few cohorts are available for such a study. By far the best option is the German Childhood Hodgkin Disease Study [11], which is a sequence of randomized clinical studies. In every subsequent study, the prescribed dose was reduced such that five different prescribed dose levels can be compared. Moreover, all treatment plans are still stored, and follow-up was up to 40 years and included routine ultrasound heart investigation. A highly significant dose-dependent increase in non-fatal but clinically significant valvular diseases and other heart diseases was reported. This source of important information should be used to study the dose dependence of one (or more) heart diseases. Similar studies could and should be performed after postoperative radiotherapy of breast cancer patients.

6. The most significant animal studies performed in recent years on the problem of the pathophysiology of radiation-induced heart disease are those conducted in Groningen on the functional interaction between radiation injury in the heart and the radiation injury of the lung [27]. They demonstrate that radiation-induced pneu- 
monitis or lung fibrosis may cause a potentially lethal increase in pulmonary hypertension, which may cause severe stress on the right heart function causing potentially lethal right heart backward failure. Any study on potentially useful predictive markers should be aware of the fact that most of the currently used heart irradiation techniques expose a larger volume of lung than heart. Radiation exposure of the lungs is known to trigger a large spectrum of molecular and biochemical responses which are only indirectly related to heart irradiation. However, this heart/lung interaction is not only a major complicating factor of radiobiological experiments on the heart, it can also be used to investigate the complex pathophysiological development of radiationinduced heart diseases by modulating the interaction of partial organ exposure of heart and lung, e. g. by varying exposed organ volumes/doses or times of radiation, thus causing functional stress during different periods of radiation damage development.

Conflict of interest K.R. Trott declares that he has no competing interests.

\section{References}

1. Cohn KE, Stewart JR, Fajardo LF et al (1967) Heart disease following radiation. Medicine (Baltimore) 46:281-298

2. Fajardo LF, Stewart JR (1970) Experimental radiation-induced heart disease. Am J Pathol 59:299-316

3. Kodama K (1993) Cardiovascular disease in atomic-bomb survivors. RERF-update

4. Carr ZA, Land CE, Kleinermann RA et al (2005) Coronary heart disease after radiotherapy for peptic ulcer disease. Int J Radiat Oncol Biol Phys 61:842-850

5. Adams MJ, Lipsitz SR, Colan SD et al (2004) Cardiovascular status in long-term survivors of Hodgkin's disease with chest radiotherapy. J Clin Oncol 22:3139-3148

6. Adams MJ, Hardenbergh PH, Constine LS et al (2003) Radiation associated cardiovascular disease. Crit Rev Oncol Hematol 43:55-75

7. Machann W, Beer M, Breunig M et al (2010) Cardiac Magnetic resonance imaging findings in 20-years survivors of mediastinal radiotherapy for Hodgkin's disease. Int J Radiat Oncol Biol Phys 76:1-7

8. Darby SC, McGale P, Taylor CW et al (2005) Long-term mortality from heart disease and lung cancer after radiotherapy for early breast cancer: prospective cohort study of about 300.000 women in US SEER registries. Lancet 6:557-565

9. Early Breast Cancer Trialists' Collaborative Group (2005) Effects of radiotherapy and of differences in the extent of surgery for early breast cancer on local recurrence and 15-year survival: an overview of the randomized trials. Lancet 366:2087-2106

10. Darby SC, Ewertz M, McGale P et al (2013) Risk of ischemic heart disease in women after radiotherapy for breast cancer. New Engl J Med 368:987-998

11. Schellong G, Riepenhausen M, Bruch C et al (2010) Late valvular and other cardiac diseases after different doses of mediastinal radiotherapy for Hodgkin diseases in children and adolescents: report from the longitudinal GPOH follow-up project oft he GermanAustrian DAL-HD studies. Pediatr Blood Cancer 55:114-1152

12. McGeoghegan D, Binks K, Gillies M et al (2008) The non-cancer mortality experience of male workers at British Nuclear Fuels plc, 1946-2005. Int J Epidemiol 37:506-518

13. Azizova TV, Muirhead CR, Drzuzhinina MB et al (2010) Cardiovascular diseases in the cohort of workers first employed at Mayak PA in 1948-1958. Radiat Res 174:155-168

14. Darby SC, Cutter DJ, Boerma M et al (2010) Radiation-related heart disease: current knowledge and future proscpects. Int J Radiat Oncol Biol Phys 76:656-665

15. Little MP, Azizova TV, Bazyka D et al (2012) Systematic review and meta-analysis of circulatory disease from exposure to lew-level ionizing radiation and etimates of potential population mortality risks. Environ Health Perspect 120:1503-1511

16. Correa CR, Das IJ, Litt HI et al (2008) Association between tangential beam treatment parameters and cardiac abnormalities after definitive radiation treatment for left-sided breast cancer. Int J Radiat Oncol Biol Phys 72:508-516

17. Taylor CW, Nisbet A, McGale P et al (2007) Cardiac exposures in breast cancer radiotherapy: 1950s-1990s. Int J Radiat Oncol Biol Phys 69:1484-1495

18. Cella L, Liuzzi R, Conson M et al (2011) Dosimetric predictors of asymptomatic heart valvular dysfunction following mediastinal irradiation for Hodgkin's lymphoma. Radiother Oncol 101:316-321

19. Taylor CW, Brönnum D, Darby SC et al (2011) Cardiac dose estimates from Danish and Swedish breast cancer radiotherapy during 1977-2001. Radiother Oncol 100:176-183

20. European Commission Directorate for Energy and Transport (2009) Emerging evidence for radiation-induced circulatory diseases. Radiation Protection 158.

21. Cardiorisk http://www.cardiorisk.eu/. Accessed 1 June 2016

22. Procardio http://www.procardio.eu/. Accessed 1 June 2016

23. Dörr W (2009) Pathogenesis of normal tissue side effects. In: Joiner M, Van der Kogel A (eds) Basic clinical radiobiology, 4th edn. vol 1. Hodder Arnold, London, pp 169-190

24. Barjaktarovic Z, Shyla A, Azimzadeh O et al (2013) Ionising radiation induces persistent alterations in the cardiac mitochondrial function of C57BL/6 mice 40 weeks after local heart exposure. Radiother Oncol 106:404-410

25. Sievert W, Trott KR, Azimzadeh O et al (2015) Late proliferating and inflammatory effects on murine microvascular heart and lung endothelial cells after irradiation. Radiother Oncol 117:376-381

26. Duma M, Herr A, Oechsner M et al (2014) Tangential Field Technique for Breast Cancer: The Dose to the Heart and Heart Subvolumes. Int J Radiat Oncol Biol Phys 90(1 Suppl):221-222

27. Van Luijk P, Faber H, Meeterns H et al (2007) The impact of heart irradiation on dose-volume effects in the rat lung. Int J Radiat Oncol Biol Phys 69:552-559 